\title{
La vinculación universidad - empresa - gobierno para la creación de spin-off universitarias de base tecnológica: el caso de una universidad pública del estado de Guanajuato
}

\section{University-company-government linkage to create technology- based university spin offs: The case of a public university from Guanajuato}

María Catalina Rangel León ${ }^{* 1}$ Universidad de Guanajuato

mc.rangelleon@ugto.mx

Rubén Molina Sánchez ${ }^{2}$ Universidad de Guanajuato rmolina@ugto.mx

Alejandra López Salazar² Universidad de Guanajuato lopez.alejandra@ugto.mx
Autor corresponsal.

1 Universidad de Guanajuato, División de Ciencias Sociales y Administrativas, Maestría en Estudios Empresariales, Ing. Javier Barros Sierra 201, Celaya, Guanajuato, MÉXICO.

2 Universidad de Guanajuato División de Ciencias Sociales y Administrativas, Departamento de Finanzas y Administración, Ing. Javier Barros Sierra 201, Celaya, Guanajuato, MÉXICO.

\section{Resumen}

Propósito: Describir la manera en que, la vinculación universidad - empresa gobierno, impulsa la creación de spin-off universitarias de base tecnológica, en una universidad pública del estado de Guanajuato.

Diseño/Metodología: La investigación es un estudio de caso con un enfoque cualitativo y un diseño etnográfico, empleando como técnica de recolección de información entrevistas a profundidad.

Resultados: Se identificó que el surgimiento de las spin-off universitarias objeto de estudio se ve impulsado por acciones individuales y conjuntas de la vinculación universidad - empresa - gobierno.

Implicaciones prácticas: Aportar pruebas respecto de las prácticas que la universidad, las empresas y el gobierno realizan, de manera individual y en conjunto, para impulsar la conformación de spin-off universitarias de base tecnológica, que sirvan como referente para ser replicadas en contextos similares.

Originalidad/Valor: Profundizar sobre el tema de la conformación de spin-off universitarias, a partir del estudio de su conformación en un contexto que puede ser aplicable en el contexto Latinoamericano.
INFORMACIÓN ARTÍCULO

Recibido: 17 de Abril 2021 Aceptado: 18 de Junio 2021

Palabras Claves:

Base tecnológica

Universidad - Empresa - Gobierno

Spin-off universitaria 


\begin{abstract}
Purpose: To describe the way in which the university-business-government linkage promotes the creation of technology-based university spin offs in a public university in the state of Guanajuato.

Design / Methodology: The research corresponds to a case study with a qualitative approach and an ethnographic design, using in-depth interviews as a data collection technique.

Results: The emergence of the university spin offs under study is driven by individual and joint actions of the university-business-government linkage. Practical implications: To provide evidence regarding the practices that the university, companies, and the government carry out, individually and jointly, to promote the creation of technology-based university spin offs, as a reference to be replicated in similar contexts.

Originality / Value: The research explores in depth the topic of university spin offs, starting with the study of their formation, which can be applicable in the Latin American context.
\end{abstract}

ARTICLE INFO

Received: 17 April 2021

Accepted: 18 June 2021

Keywords:

Technological base

University - Business - Government

University spin-off

\section{INTRODUCCIÓN}

El papel de la universidad en la economía del conocimiento actual ha sido reconocido desde hace mucho tiempo y se ha reafirmado en variados estudios (Rosemberg y Nelson, 1994; Jacob, 2000; Etzkowitz, 2004; Martínez y Corrales, 2011). Ejemplo de lo anterior, el papel que han jugado universidades como Stanford, Massachusetts Institute of Technology y Cambridge, para la creación del Silicon Valley como desarrollo de la región de Boston y el crecimiento de las telecomunicaciones en Reino Unido.

No obstante, los nuevos conocimientos deben ser incorporados a las llamadas técnicas aplicadas, pues por sí mismos, aun cuando permiten obtener reconocimiento científico, carecen de relevancia cuando se habla de valor económico (Degroof y Roberts, 2004; Clark, 2004). Muestra de dichas técnicas la conforman las llamadas spin-off universitarias, referidas a una nueva empresa creada bajo el amparo de una universidad, con el objeto de explotar sus resultados de investigación traducidos en productos y servicios avanzados (Pirnay, 1998).

El emprendedor o el grupo de emprendedores generalmente corresponde a personal universitario como profesores, investigadores o alumnos, y estos últimos con nivel de posgrado (Ndonzuau, Pirnay y Surlemont, 2002; Rubio, 2009). Por lo anterior, resulta trascendente aclarar que el concepto de spin-off se refiere a nuevas actividades económicas derivadas de otra empresa o entidad ya existente que, con su apoyo, adquieren independencia y viabilidad propia en términos de estructura jurídica, técnica y comercial. Las empresas o entidades de las que surgen funcionan como matriz, apoyando en el despegue de la spinoff (Simón, 2003).

Debido a que en el actual contexto mundial se demandan mejores productos y servicios, los paises han debido implementar estrategias para ofrecer productos y servicios avanzados o de valor agregado con la finalidad de generar riqueza (Von Graevenitz, Harhoff y Weber, 2010). Es así como la conformación de spin-off universitarias constituye una de dichas estrategias, porque favorece la aplicación del conocimiento, la transferencia de tecnología, la innovación, la generación de empleo y, por ende, el desarrollo industrial (Rodríguez-Gulías, Fernández-López y Rodeiro-Pazos, 2016; Mathisen y Rasmussen, 2019).

Con lo anterior, la vinculación universidad - empresa - gobierno, representada en el modelo de la triple hélice, juega un papel importante pues sienta las bases para detonar innovaciones tecnológicas en el entorno, a través de la red de interacciones creadas entre los actores, a partir de la conjunción de esfuerzos para maximizar el valor de las actuaciones individuales, creando un escenario para la conformación de spin-off universitarias de base tecnológica (Cai, 2014; Cai y Lattu, 2019; Samo y Huda, 2019; Gabrielsson, Politis y Billström, 2019). 
De esta forma, la presente investigación busca responder a la pregunta de investigación ¿de qué manera la vinculación universidad - empresa gobierno, impulsa la creación de spin-off universitarias de base tecnológica en una universidad pública del estado de Guanajuato? La originalidad de la investigación se basa en lo poco estudiado del tema y que la universidad objeto de estudio se encuentra ubicada en el ranking de las primeras 15 universidades en México (Sáez, 2018), además, para 2020 ubicada entre las 100 mejores universidades de Latinoamérica de acuerdo con el Ranking Latinoamericano de Universidades QS (QS Quacquarelli Symonds Limited, 2021), lo que abonaría a la literatura para la identificación de prácticas que impulsen la conformación de spinoff universitarias en contextos similares.

En lo que se refiere a la estructura del presente, el texto se divide en cinco secciones: en la primera se presenta la revisión de literatura, enseguida se detalla la metodología, en la subsecuente se presentan los resultados y la discusión y, por último, se presentan las conclusiones.

\section{REVISIÓN DE LITERATURA}

\section{Modelo de innovación de la triple hélice para conformación de spin-off universitarias}

Dentro de la literatura existente sobre modelos de innovación, aquel que cuenta con un mayor grado de aceptación por la comunidad científica corresponde al modelo de triple hélice propuesto por Etzkowitz y Leydersdorff (1995), estudiado posteriormente por Jones-Evans y Klofsten (1997) y más recientemente por Numprasertcha y Igel (2005); Cai (2014); Cai y Lattu (2019); Samo y Huda (2019) y Gabrielsson y cols., (2019). Este modelo estudia el actuar de manera individual y las interacciones entre tres diferentes agentes o esferas: la universidad, la empresa y el gobierno.

Sobre lo anterior, se ha confirmado la necesidad de conjuntar los esfuerzos de cada uno de los agentes con la finalidad de multiplicar el valor de sus funciones individuales, por ejemplo, se visualiza a las empresas como demandantes de innovaciones tecnológicas, traducidas en productos y servicios tecnológicos que les permitan optimizar sus procesos e incrementar su competitividad. Por otro lado, se identifica el papel de las universidades como generadoras de nuevo conocimiento, traducido en investigaciones y posibles invencio- nes que podrían servir como oferta tecnológica para atender los requerimientos empresariales (Cai y Lattu, 2019; Samo y Huda, 2019 y Gabrielsson y cols., 2019). Aun cuando la relación entre universidades y empresas pareciera imperativa, no ha sido así. En la actualidad sigue siendo necesaria una reestructuración para dar lugar a dicha vinculación (Montiel-Campos, 2018).

Específicamente, en el modelo III de la triple hélice cada uno de los agentes o esferas se encuentra vinculado e interrelacionado, lo que le permite generar los llamados efectos positivos, mismos que le permiten tener repercusiones sobre los tres, por el llamado efecto de reciprocidad $y$, por consiguiente, para la sociedad en conjunto. Dichos efectos positivos incrementan el valor de sus funciones individuales y, en conjunto, generan la denominada espiral del conocimiento, que impulsa procesos de I+D+i (Etzkowitz y Leydesdorff, 1995, 2000; Cai 2014). Asimismo, se incluyen en el modelo los denominados agentes híbridos de innovación, conformados por instituciones que facilitan la vinculación entre las esferas, como los parques tecnológicos y científicos o los promotores tecnológicos (Amoroso, Link y Wright, 2019).

\section{El papel de la universidad: la universidad emprendedora}

En el modelo y derivado de la misión académica y científica de la universidad, esta se convierte en un eslabón clave dentro de los sistemas de innovación, dada la importancia de la producción de conocimiento y su aplicación en la atención de necesidades de la industria, lo que favorece un cambio en su enfoque y énfasis en su función como universidad emprendedora (Clark, 2004; Etzkowitz, 2004; Gibb, 2005).

La universidad emprendedora es referida como una institución proactiva en la aplicación del conocimiento generado y que opera bajo un modelo de innovación interactivo, partiendo de la identificación de necesidades en la industria y la satisfacción de estas por medio de la ciencia (Amoroso y cols., 2019). Resultado de lo anterior, la universidad necesita realizar innovaciones organizacionales, nuevos acuerdos con la sociedad y crear nuevas vías de comunicación con el objetivo de cumplir con esa nueva función, actuar como un actor económico por sí misma y adaptarse a los cambios en sus contextos (Vesperi, Fatta y Parra, 2018).

Es así como la universidad, en su rol de promover 
la actividad de $1+\mathrm{D}+\mathrm{i}$, tiene como mecanismo la creación de spin-off universitarias como una pieza intermediaria entre los intereses académico-científicos y comerciales demandados por el mercado. Las spin-off universitarias se convierten en un punto de contacto entre ambas realidades. De ahí su notoriedad como herramienta estratégica del sistema público de $1+D+i$ y de la sociedad en su conjunto (Buratti, Profumo y Persico, 2020).

\section{El papel del sector empresarial}

Dentro del modelo, a las empresas, en el cumplimiento de su misión que consiste en la satisfacción de necesidades del mercado, les resulta imprescindible la incorporación de innovaciones resultado de proceso de $\mathrm{I}+\mathrm{D}+\mathrm{i}$, derivado de la actual dinámica económica y la intensidad de rivalidad entre competidores (Buratti y cols., 2020). Sin embargo, en el grueso de los casos, el realizar actividades de $1+D+i$ representan altos costos de inversión, por lo que es más viable la incorporación de innovaciones creadas por terceros, aunque la investigación sea realizada por la empresa. El acercamiento con las universidades se releva y permite tener acceso a productos y servicios de base tecnológica, como la asesoría, consultoría y la utilización de equipos o infraestructura especializada (Amoroso y cols., 2019).

\section{El papel del gobierno}

Dentro del modelo el gobierno cuenta con una posición conciliadora de los intereses de cada uno de los agentes o esferas, ello derivado de su misión por lograr un crecimiento económico y desarrollo social, a través del desarrollo e incorporación de innovaciones (Vega-Gómez, Miranda y Pérez-Mayo (2020). De acuerdo con Julien y Molina (2012), el rol del estado puede resumirse en cinco funciones principales, a saber: 1) apuntalar, consiste en conocer en detalle las necesidades de las regiones, para a partir de ellas direccionar los esfuerzos hacia los sectores que así se definan, 2) conectar, consiste en ayudar a desarrollar redes complejas para vincular a emprendedores, 3) sostener, consiste en apoyar a las empresas más distintivas o proactivas de la región, con ayudas complementarias, 4) estimular el tejido industrial multiplicando los lugares de apoyo y desarrollo de la I+D y facilitando los vínculos entre estos y las empresas, 5) facilitar el aprendizaje de los actores con el fin de multiplicar las ideas.

\section{METODOLOGÍA}

La estrategia de investigación utilizada fue el estudio de caso (Yin, 1989) con un enfoque cualitativo (Enríquez, 2017) y un diseño etnográfico (Hernández y Mendoza 2018), que permite describir la manera en que la vinculación universidad - empresa - gobierno impulsa la creación de spin-off universitarias de base tecnológica de una universidad pública del estado de Guanajuato, a partir de cuatro categorías de análisis.

\section{Muestra}

De acuerdo con Hernández y Mendoza (2018), las unidades de muestreo para la investigación fueron organizaciones, grupos y personas. Las unidades de análisis correspondieron a prácticas, procesos y episodios. Los factores que determinaron el número de casos y los recursos con los que se dispuso, corresponden al número total de casos identificados. El tipo de muestra fue por conveniencia y casos tipo, debido a que se entrevistó al total de casos identificados y a los que se tuvo acceso, buscándose riqueza, profundidad y calidad en la información (Battaglia, Sampling y Lavrakas, 2008).

El tamaño de la muestra correspondió a ocho spin-off universitarias de base tecnológica industriales y de servicios en las áreas de eficiencia energética, energías renovables, TIC's, genética molecular de hongos, materiales avanzados y poliestireno expandido (EPS). Cada una de ellas con figura jurídica independiente a la universidad, pero surgidas de forma planeada, con el objetivo de comercializar una tecnología o conocimiento generado al interior de la universidad, traducido en un registro de propiedad industrial. El titular de la empresa es o fue el inventor/emprendedor y trabaja o trabajó en la universidad desempeñándose como profesor de tiempo completo o estudiante de posgrado. Entre los tipos de estructura financiera se consideran asociados, joint venture o propia.

Adicional a las ocho spin-off universitarias, se realizaron tres entrevistas a empleados de instituciones gubernamentales encargados de temas sobre tecnología, investigación, desarrollo e innovación del estado de Guanajuato y cuatro entrevistas a personal universitario en áreas de vinculación, extensión, proyectos y apoyo a la investigación, con la finalidad de lograr una triangulación de los datos. De acuerdo con Hernández y Mendoza (2018), 
el número de casos se encuentra dentro del rango del tamaño de muestra sugerido para un estudio de caso. El período de levantamiento de datos fue del 14 de febrero al 21 de agosto de 2020.

\section{Herramienta de recolección de información}

Como técnica de recolección de información se utilizó la entrevista en profundidad (Vasilachis de Gialdino, 2009), y para ello se elaboró un guion de entrevista mediante una matriz de autores que permitió definir categorías y propiedades de análisis iniciales que condujeran el proceso de entrevista.

\section{Estrategia para análisis de los datos}

Para el análisis de los datos obtenidos se utiliza como estrategia el desarrollo de una narrativa general en donde se incluyeron las unidades de análisis, categorías (conformadas mediante la codificación abierta y axial), temas y patrones emergentes de los datos. Lo anterior a través de la realización de descripciones detalladas y la presentación de experiencias que fueron contrastadas con la teoría para la construcción de significados. Para lo descrito, se utilizó el software Atlas.ti versión 7, mediante la construcción de una unidad hermenéutica a través de la codificación abierta línea por línea para relacionar y fusionar códigos, logrando la codificación axial de los datos obtenidos, la construcción de categorías, temas y familias de códigos gracias a la saturación de cada uno de ellos durante el análisis y su posterior soporte con la teoría (Weitzman y Miles, 1995).

\section{RESULTADOS Y DISCUSIÓN}

Los hallazgos obtenidos del estudio de campo fueron clasificados en cuatro familias de códigos que correspondieron a la universidad, las empresas, el gobierno y la espiral del conocimiento. De dichas familias se desprendió un total de treinta y cinco códigos. A continuación, se presenta cada una de las familias de códigos.

\section{Universidad}

Sobre la base de las entrevistas realizadas a personal universitario, se identificó que la universidad ha tenido que realizar innovaciones organi- zativas graduales, con la finalidad de modificar prácticas arraigadas entre su personal académico, respecto a la aplicabilidad del nuevo conocimiento generado en cada una de las investigaciones realizadas. Es así como han trabajado en la detección de necesidades en la industria para proponer soluciones en la ciencia con un modelo de innovación interactivo, lo que concuerda con lo sugerido por Vesperi, Fatta y Parra (2018), quienes identifican la existencia de desafíos económicos y sociales para las universidades, por lo que, deberán realizar innovaciones organizacionales que les permitan adaptarse a los cambios en sus contextos.

Por lo anterior, la universidad ha debido realizar acercamientos con el sector industrial, materializado en la celebración de convenios de colaboración, donde han buscado la vinculación con el gobierno. Resultado de los nuevos acuerdos sociales realizados por la universidad, esta ha tenido que crear estrategias para trasladar el conocimiento (traducido en productos y servicios tecnológicos) hacia la industria, mismas que la han llevado a fungir como un actor económico por sí misma, a partir de la comercialización de los resultados de investigación desde diversos mecanismos.

Entre los mecanismos destacan: la celebración de convenios de colaboración con los sectores empresarial o gubernamental, la celebración de contratos de servicios profesionales y especializados, el licenciamiento de patentes, la movilidad de recursos humanos entre la universidad y la empresa, las publicaciones en revistas y aportaciones a eventos de divulgación y finalmente, la creación de empresas universitarias, entre las que se encuentran spin-off universitarias de base tecnológica. Sin embargo, estas no representan la principal estrategia o la que mejores resultados les ha generado. Véase figura 1.

\section{Empresa}

Como parte del papel que debe cubrir el sector industrial respecto a la satisfacción de necesidades en el mercado, se encontró preocupación de los titulares de tres de las spin-off universitarias entrevistadas, por la intensa rivalidad entre competidores en la que se encuentran inmersos. Dicha rivalidad los ha orillado a incorporar procesos de I+D+i para la creación de innovaciones organizacionales, sea para hacer más eficientes los procesos y generar mayores beneficios económicos a la empresa y, por ende, ser más competitivos en costos, o bien, incorporadas en los productos 


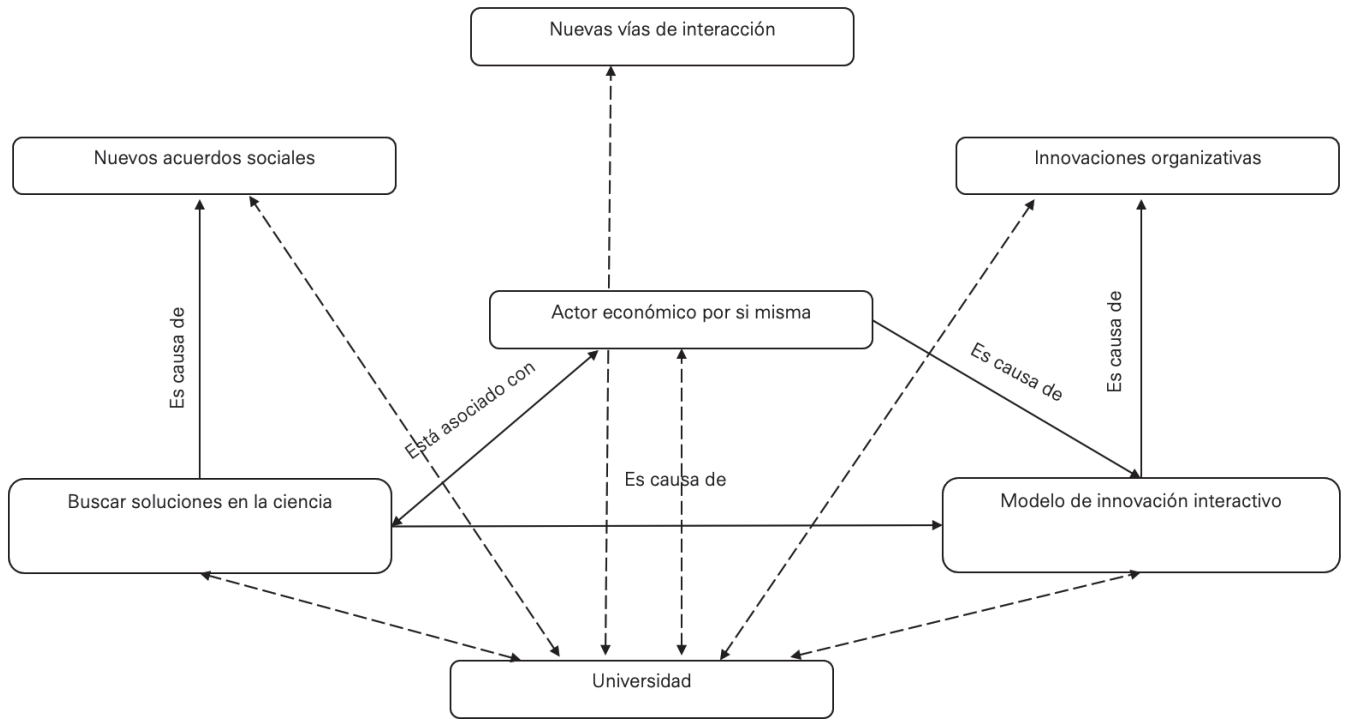

Figura 1: Familia de códigos: universidad. Fuente: Elaboración propia.

y servicios para darles un valor agregado. Sin embargo, en la mayoría de las ocasiones, dichas medidas representan altos costos de inversión por la necesidad de contratación de personal especializado en el área o por la necesidad de adquisición de infraestructura, equipo y/o maquinaria especializada, lo que incide en el tiempo que se destinaría a actividades previamente definidas.

Ante tal situación, tres spin-off universitarias entrevistadas han debido optar por la vinculación con la universidad, situación que las ha llevado a disminuir los costos de inversión al adquirir directamente los servicios que dos de las cinco spin-off universitarias entrevistadas ofrecen. Lo anterior concuerda con lo que describen Buratti, Profumo y Persico (2020), quienes resaltan las necesidades de mercado como uno de los factores que incentiva la conformación de spin-off universitarias. Véase figura 2.

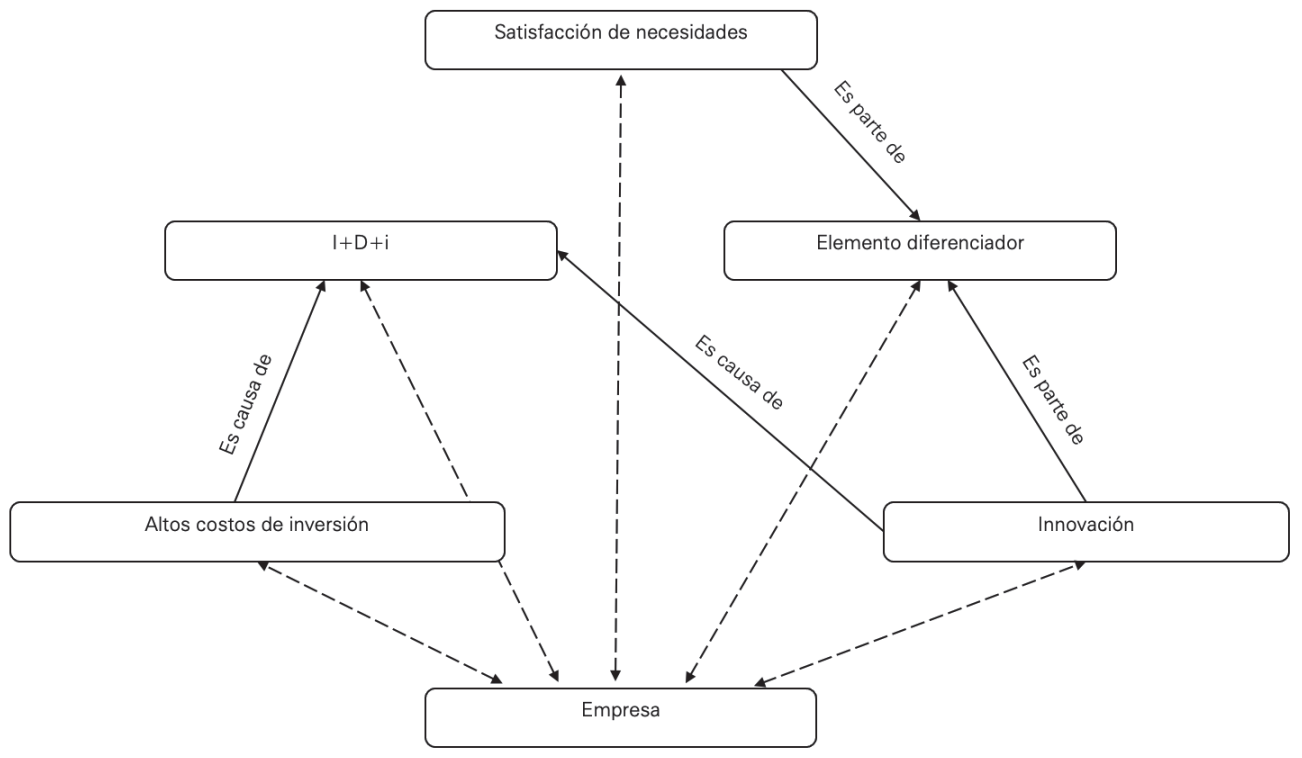

Figura 2: Familia de códigos: empresa. Fuente: Elaboración propia. 


\section{Gobierno}

Las acciones encontradas como parte del rol del gobierno se agruparon en cinco categorías: 1) apuntalar, 2) conectar, 3) sostener, 4) estimular y 5) facilitar, lo que coincide con lo propuesto por Julien y Molina (2012). Respecto a la categoría de apuntalar, se encontraron actividades del gobierno estatal en conjunto con el gobierno federal por medio del Consejo Nacional de Ciencia y Tecnología (CONACYT), orientadas a la identificación de necesidades, vocaciones económicas y capacidades del estado de Guanajuato plasmadas en la agenda de innovación del estado. Lo expresado concuerda con lo planteado por Vega-Gómez, Miranda y Pérez-Mayo (2020), quienes identifican la existencia de políticas públicas que promuevan la conformación de spin-off universitarias.

Como parte del rol de conectar, el estado se da a la tarea de fomentar redes complejas a través de la promoción para la creación de un ambiente de innovación identificado como el ecosistema de innovación del estado de Guanajuato, conformado por: instituciones de educación, centro de investigación, parques tecnológicos, promotores tecnológicos, incubadoras y aceleradoras de negocios, oficinas de transferencia de tecnología o del co- nocimiento (OTT/OTC), laboratorios nacionales, cámaras empresariales, clústeres y programas de apoyo. Dicho ecosistema coincide con lo sugerido por Vega-Gómez y cols. (2020), quienes identificaron la existencia de un ambiente que promueve la formación de los titulares y colaboradores de las spin-off como factor que motiva la conformación de nuevas spin-off universitarias y su supervivencia a largo plazo.

En este último sentido, los programas de apoyo forman parte del tercer rol del estado que corresponde a la categoría de sostener, traducido en apoyos económicos estatales para el desarrollo y aplicación del conocimiento científico y tecnológico en las áreas de especialización y sectores prioritarios determinados. En la quinta categoría, el gobierno facilita el aprendizaje entre las esferas institucionales a partir de la creación de la legislación en materia de investigación científica, tecnológica y de innovación a través de la Ley de Fomento a la Investigación Científica, Tecnológica y a la Innovación para Guanajuato, sentando las bases para el Sistema de Innovación y fomentando la atracción, creación y desarrollo de empresas de base tecnológica. Véase figura 3.

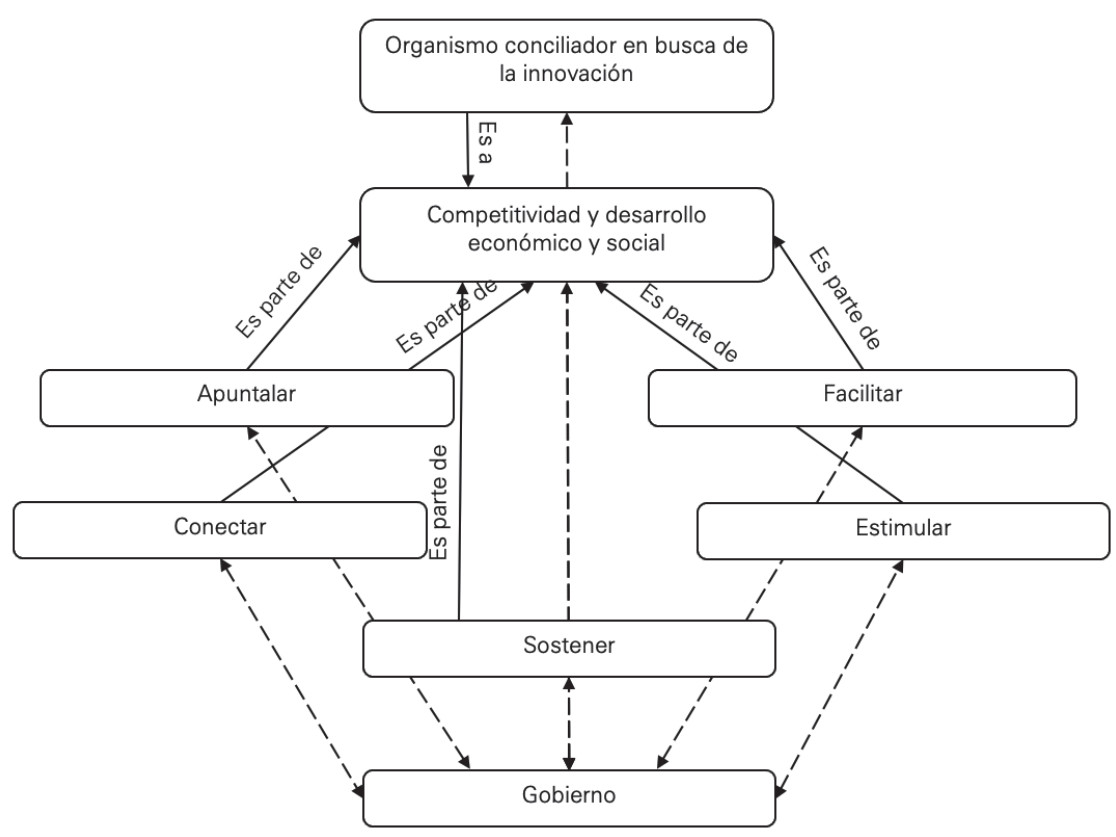

Figura 3: Familia de códigos: gobierno. Fuente: Elaboración propia. 


\section{La espiral del conocimiento}

Habiendo presentado los resultados de los roles de la universidad, las empresas y el gobierno, a continuación, se presentan los resultados sobre la manera en que las relaciones que guarda cada uno de los elementos a través de acciones individuales y en conjunto, impulsan la conformación de spin-off universitarias de una universidad pública del estado de Guanajuato. Los resultados se obtienen a partir de la identificación de actividades complementarias entre cada una de las esferas institucionales, porque de acuerdo con los testimonios, ante la necesidad del sector industrial por disminuir los altos costos de inversión requeridos para realizar procesos de $\mathrm{I}+\mathrm{D}+\mathrm{i}$ que les permitan generar innovaciones, han debido reali- zar vinculación con dos de las spin-off universitarias entrevistadas. Estas últimas impulsadas en su creación por el ecosistema de innovación del estado de Guanajuato.

Como se presentó, en lo concerniente a la espiral del conocimiento, se identificó la existencia de dicho fenómeno, sin embargo, el mismo no ha formado parte de la conformación de las spinoff objeto de estudio. No obstante, siguiendo a Prokop, Huggins y Bristow (2019), las spin-off universitarias dependen de las conexiones de las redes para acceder a determinados recursos, por lo que la espiral del conocimiento existente en el contexto estudiado podría motivar el surgimiento de nuevos emprendimientos. Véase figura 4.

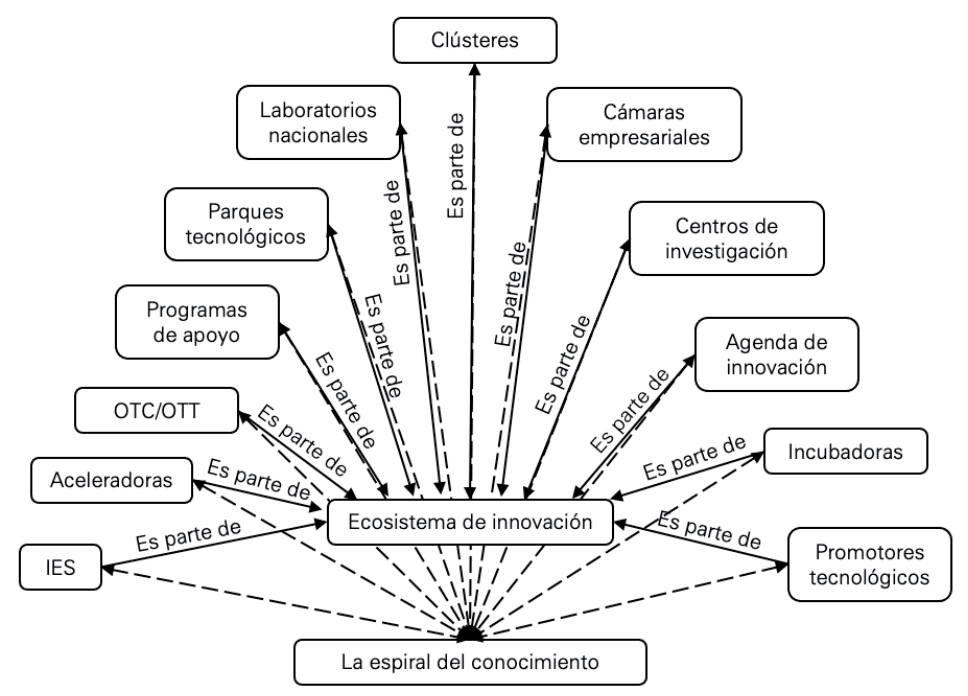

Figura 4: Familia de códigos: la espiral del conocimiento. Fuente: Elaboración propia.

\section{CONCLUSIONES}

Respecto al objetivo de la investigación, se identificaron actividades individuales e interrelaciones entre la universidad, la empresa y el gobierno como impulsores para la creación de spin-off universitarias en la universidad objeto de estudio.

Por parte de la universidad, se destaca la nece200 sidad de adaptarse a los nuevos requerimientos sociales que demandan la aplicación del conocimiento generado al interior de las universidades en los temas prioritarios del estado. Lo anterior, llevó a la institución a realizar reestructuras organizativas que promovieran la incorporación de una nueva función: la de emprendimiento, sin que ello representara el descuido de su función sustantiva de enseñanza, así como la de investigación. 
Para ello, se incorpora a lo largo del tiempo instancias, programas y normatividad orientada hacia los requerimientos sociales desde la oferta de programas educativos en ciencias e ingenierías, fomento de la calidad de sus investigadores, creación de instancias y normatividad que propicie el desarrollo de proyectos para el sector empresarial y gubernamental (como OEl's, incubadoras de negocios, parques tecnológicos, áreas de vinculación, OTT's), lo que promueve un ambiente de emprendimiento como uno de los elementos para trasferir los resultados de investigación. De esta forma, las incorporaciones se convierten en parte de las implicaciones prácticas de la presente investigación.

Asimismo, en las empresas se encontró la necesidad de incorporar I+D en sus procesos, permitiéndoles realizar innovaciones con la finalidad de lograr una posición de mayor ventaja en el sector de actividad económica en el que se encuentre compitiendo, sin embargo, dichos requerimientos les representan elevados costos de inversión. Con lo anterior, el sector empresarial demanda que las universidades puedan atender dicha necesidad a través de las investigaciones realizadas.

Finalmente, derivado de uno de los objetivos principales del gobierno, que consiste en generar crecimiento económico y desarrollo social de las regiones para volverlas competitivas, el gobierno cubre el papel de impulsar, regular y sostener las relaciones entre universidades y sector empresarial con la finalidad de ofertar bienes y servicios con un valor agregado, resultado del conocimiento generado al interior de las universidades. Por lo que se identificó con la presente investigación la existencia de un ecosistema de innovación en el estado de Guanajuato conformado por distintos actores identificados como: OTT's, parques tecnológicos, laboratorios nacionales, aceleradoras e incubadoras de negocios, IES, centros de investigación, clústeres, cámaras empresariales y promotores tecnológicos, programas de financiamiento que promueve la $\mathrm{I}+\mathrm{D}+\mathrm{i}$, que se traduce en emprendimientos de alto impacto que atienden a las necesidades universitarias y del sector productivo. Este ecosistema constituye parte de las implicaciones prácticas de la investigación, constituyéndose como objeto de análisis en caso de ser replicado en contextos semejantes.

Por lo anterior, con base en las limitaciones de la presente investigación al tratarse de un estudio de caso, se proponen como futuras líneas de in- vestigación el análisis de los diferentes modelos de innovación (como penta hélice e innovación abierta), para impulsar la creación de spin-off universitarias en diferentes centros generadores del conocimiento, como otras universidades y centros de investigación. Se propone, además, analizar los diversos mecanismos de traslado del conocimiento hacia el sector productivo para identificar áreas de oportunidad en la diversificación de financiamiento de las universidades y los centros de investigación.

\section{BIBLIOCRAFIA}

Amoroso, S., Link, A., y Wright, M. (Eds.). (2019). Science and Technology Parks and Regional Economic Development: An International Perspective. Springer Nature.

Battaglia, M., Sampling, N., y Lavrakas, P. (2008). Encyclopedia of survey research methods. Publication date.

Buratti, N., Profumo, G., y Persico, L. (2020). The impact of market orientation on university spin-off business performance. Journal of International Entrepreneurship, 1-26.

Cai, Y. (2014). Implementing the Triple Helix model in a non-Western context: an institutional logics perspective. Triple Helix, 1(1), 1-20.

Cai, Y., y Lattu, A. (2019, September). Civically grounded Triple Helix: Synergies between Triple Helix and Quadruple Helix models of innovation. In Proceedings of the XVII International Triple Helix Conference, Cape Town, South Africa, 9-11.

Clark, B. (2004). Sustaining Change in Universities. Society for Research into Higher Education. Open University Press. Londres.

Degroof, J. y Roberts, E. (2004). Overcoming weak entrepreneurial infrastructures for academic spin-off ventures. Journal of Technology Transfer, 29, 327-352.

Enríquez, M. (2017). Etnografía y observación participante en investigación cualitativa de Michael Angrosino. Maguaré, 31(2), 258-262.

Etzkowitz, H. (2004). The evolution of the entrepreneurial university. International Journal of Technology and Globalisation, 1(1), 64-77. 
Etzkowitz, H., y Leydesdorff, L. A. (1995). Universities and the global knowledge economy: $A$ triple helix of university-industry-government relations. Recuperado de: https://hdl.handle. net/11245/1.115517

Etzkowitz, H., y Leydesdorff, L. (2000). The dynamics of innovation: from National Systems and "Mode 2" to a Triple Helix of universityindustry-government relations. Research policy, 29(2), 109-123.

Gabrielsson, J., Politis, D. y Billström, A. (2019). University spin-offs and triple helix dynamics in regional innovation ecosystems: a comparison of technology intensive start-ups in Sweden. Global Business and Economics Review, 21(3-4), 362-381.

Gibb, A. (2005). Towards the entrepreneurial university: entrepreneurship education as a lever for change. Policy paper, 3, 1-46.

Hernández-Sampieri, R., y Mendoza, C. (2018). Metodología de la investigación. Las rutas cuantitativa, cualitativa y mixta. México: McGraw Hill.

Jacob, M. (2000). Mode 2 in context: The contract researcher, the university and the knowledge society. The future of knowledge production in the academy and open university press, 1127.

Jones-Evans, D. y Klofsten, M. (1997). Universities and local economic development: The case of Linköping. European Planning Studies, 5(1), 77-93.

Julien, P. A., y Molina, R. (2012). Una teoría sobre el emprendimiento regional en la economía del conocimiento: una metáfora de las novelas policiacas. Pearson.

Martínez, A. y Corrales, M. (2011). Administración de Conocimiento y desarrollo basado en conocimiento. Redes e innovación. México. Cengage Learning.

Mathisen, M. y Rasmussen, E. (2019). The development, growth, and performance of university spin-offs: a critical review. The Journal of Technology Transfer, 44(6), 1891-1938.

Montiel-Campos, H. (2018). University spin-offs creation in the Latin American region. Journal of Entrepreneurship in Emerging Economies.
Ndonzuau, F. N., Pirnay, F., y Surlemont, B. (2002). A stage model of academic spin-off creation. Technovation, 22(5), 281-289.

Numprasertchai, S. e Igel, B. (2005). Managing Knowledge trough collaboration: multiple case studies of managing research in university laboratories in Thailand. Technovation, 25, 1173-1182.

Pirnay, F. (1998). Spin-off et essaimage: de quoi s' agit-il? Une revue de la littérature. In Compétitivité et identité des PME. Recuperado de: http://pascal-francis.inist.fr/vibad/index. php?action $=$ getRecordDetail\&idt $=6204817$

Prokop, D., Huggins, R., y Bristow, G. (2019). The survival of academic spinoff companies: An empirical study of key determinants. International Small Business Journal, 37(5), 502-535.

QS Quacquarelli Symonds Limited (23 de junio de 2021). QS Latin America University Rankings 2021. Recuperado de https://www.topuniversities.com/university-rankings/latin-american-university-rankings/2021

Rodríguez-Gulías, M., Fernández-López, S., y Rodeiro-Pazos, D. (2016). Growth determinants in entrepreneurship: A longitudinal study of Spanish technology-based university spinoffs. Journal of International Entrepreneurship, 14(3), 323-344.

Rosemberg N. y Nelson, R. (1994). American Universities and Technical Advance in Industry, Research Policy, 23(3), 323-348.

Rubio, A. (2009). Spin-off, la fuerza centrífuga de las ideas Centros europeos de empresas innovadoras (CEEl-Valencia). Recuperado de: http://www.ceei-valencia.com/index.php

Sáez, C. (2018). Ranking de universidades México 2018. MBA and Education. Recuperado de: https://mba.americaeconomia.com/articulos/ notas/ranking-de-universidades-de-mexico-2018

Samo, A. y Huda, N. (2019). Triple Helix and academic entrepreneurial intention: understanding motivating factors for academic spin-off among young researchers. Journal of Clobal Entrepreneurship Research, 9(1), 1-15.

Simón, E. (2003). La creación de empresas de base tecnológica: Una experiencia práctica. Proyecto para la promoción de empresas innovadoras de base tecnológica. España. 
Vasilachis de Gialdino, Irene (2009). Los fundamentos ontológicos y epistemológicos de la investigación cualitativa. Forum Qualitative Sozialforschung / Forum: Qualitative Social Research, 10(2),

Vega-Gómez, F. I., Miranda González, F. J., y Pérez-Mayo, J. (2020). Analyzing the Effects of Institutional-and Ecosystem-Level Variables on University Spin-Off Performance. SAGE Open, 10(2), 1-14.

Vesperi, W., Fatta, D., y Parra, C. (2018). Providing a general framework about spin-off success factors in complex environments. International Journal of Markets and Business Systems, 3(2), 93-110.
Von Graevenitz, G., Harhoff, D. y Weber, R. (2010). The effects of entrepreneurship education. Journal of Economic Behavior y Organization, 76(1), 90-112.

Weitzman, E. y Miles, M. (1995). Computer programs for qualitative data analysis. Sage.

Yin, R. (1989). Case Study Research: Design and Methods, Applied social research Methods Series, Newbury Park CA, Sage. 
Revista Academia \& Negocios Vol.7 (2) 2021 pp. 193-204 